\title{
Extraluminale Valvuloplastie - Indikation und Ergebnisse anhand eines Fallberichtes
}

\section{Mühlberger ${ }^{1,2} ;$ A. Mumme ${ }^{1,2} ;$ S. Reich-Schupke 2 ;. Hummel ${ }^{1,2}$}

${ }^{1}$ Klinik für Gefäßchirurgie, Katholisches Klinikum Bochum, Klinikum der Ruhr Universität Bochum; ${ }^{2}$ Venenzentrum der Dermatologischen und Gefäßchirurgischen Kliniken, Ruhr Universität Bochum

\begin{abstract}
Schlüsselwörter
Extraluminale Valvuloplastie, V. saphena magna, pAVK, Bypasschirurgie

Zusammenfassung

Die meisten der zur Verfügung stehenden Verfahren zur Sanierung einer Stammvarikose arbeiten zerstörend oder entfernend. Dies gilt sowohl für die operative Entfernung, endovenöse Katheterverfahren als auch die Sklerotherapie. Als venenerhaltende Verfahren der V. saphena magna (VSM) wurden lediglich die extraluminale Valvuloplastie, das ASVAL Verfahren sowie die CHIVA Methode beschrieben. 10 Jahre nach Durchführung einer extraluminalen Valvuloplastie konnte in unserem Hause die VSM nun erstmals als Bypassgefäß zur Anlage eines femoro-poplitealen Bypass bei einer pAVK verwendet werden. Dabei zeigte sich duplexsonographisch und intraoperativ eine gut geeignete Vene ohne postphlebitische Veränderungen.
\end{abstract}

\section{Keywords}

Valvuloplasty, great saphenous vein, PAOD, vascular surgery

\section{Summary}

Frequently used treatment methods for great saphenous vein insufficiency e.g. endovenous or surgical therapy, result to a destruction or removal of the vein. There exist only several methods (e.g. ASVAL or CHIVA method or external valvuloplasty) which lead to a preservation of the great saphenous vein. In this case report we describe a patient who had a femoro-popliteal bypass surgery. The great saphenous vein of the contralateral leg, which was successfully treated by external valvuloplastiy 10 years ago, was uses as a homograft. Intraoperatively the vein did not show any pathological changes.

External valvuloplasty - indications and results based on a case report Phlebologie 2018; 47: 257-260 https://doi.org/10.12687/phleb2439-5-2018 Eingegangen: 27. Juni 2018 Angenommen: 11. Juli 2018

English version available at: www.thieme.de/phlebo

\section{Einleitung}

Die meisten der zur Verfügung stehenden Verfahren zur Sanierung einer Stammvarikose arbeiten zerstörend oder entfernend (1). Dies gilt sowohl für die operative Entfernung, endovenöse Katheterverfahren als auch die Sklerotherapie (2). Als venener- haltende Verfahren der VSM wurden lediglich die extraluminale Valvuloplastie, das ASVAL Verfahren sowie die CHIVA Methode beschrieben $(1,3-6)$. Von diesen scheint die extraluminale Valvuloplastie derzeit in Deutschland am häufigsten angewendet zu werden (1). Grundlage der extraluminalen Valvuloplastie ist die patho- physiologische Theorie der „deszendierenden Varikose“ (7). Man geht davon aus, dass eine Inkompetenz der terminalen bzw. präterminalen Klappe in frühen Stadien der Erkrankung auftritt und es erst in der Folge $\mathrm{zu}$ einer Schädigung distaler $\mathrm{Ab}$ schnitte kommt $(2,8)$. Durch die Implantation eines Kunststoffpatches um die Vene im Bereich der saphenofemoralen Mündung kommt es zu einer Reduktion des Venendurchmessers auf ein physiologisches $\mathrm{Maß}$ von ca. $5 \mathrm{~mm}$ und somit zu einer wiederhergestellten Funktionsfähigkeit der terminalen bzw. präterminalen Klappe (9). Dadurch sistiert der Reflux und in weiterer Folge kann es auch zu einer Retonisierung der übrigen VSM kommen (5). Der Erhalt der VSM ist die Grundlage für eine spätere Verwendung der VSM als Bypassgefäß für aortokoronare oder femoro-popliteale $\mathrm{Re}$ konstruktionen. Dabei ist eine funktionsfähige VSM entscheidend für den späteren Outcome. So sind beispielsweise die Offenheitsraten bei Verwendung der VSM anstatt eines Kunststoffmaterials deutlich höher (10). Zudem ist venöses Material, im Gegensatz zu Kunststoffmaterial, auch im Fall einer Infektion oder Entzündung Mittel der Wahl.

Voraussetzung für die Indikationsstellung einer extraluminalen Valvuloplastie ist jedoch eine detaillierte Duplexsonographie. So sind beispielsweise Avalvulie, postphlebitische Veränderungen der VSM oder der Venenklappen sowie ein Durchmesser der Vene von $>10 \mathrm{~mm}$ bei Frauen bzw. 12 mm bei Männern eine Kontraindikation zur Anwendung des Verfahrens.

Besonders geeignete Indikationen für die Anwendung einer extraluminale Vavuloplastie sind (9):

- Stammveneninsuffizienz der V. saphena magna im Stadium I nach Hach 


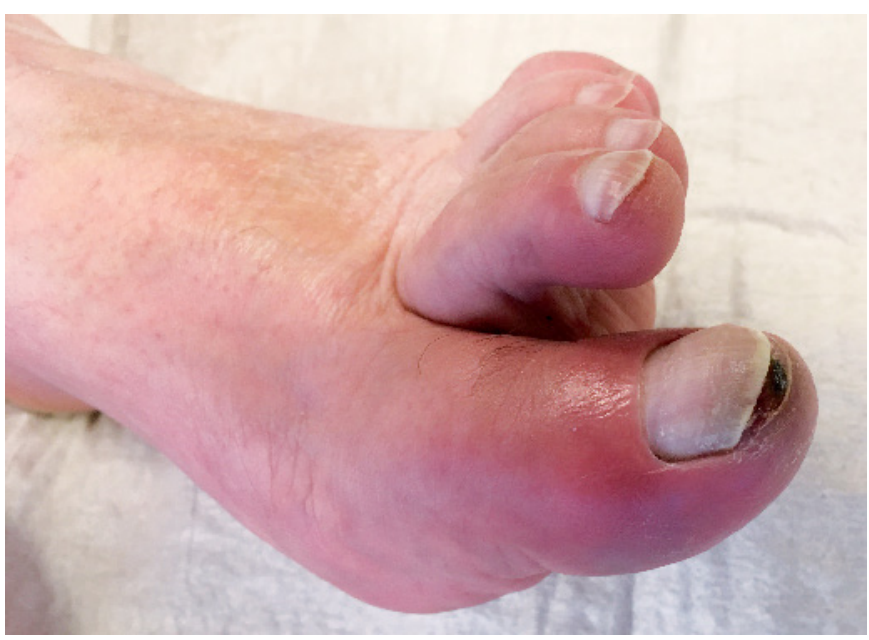

Abb. 1

Bagatelltrauma der Großzehe mit beginnender Rötung des Vorfußes

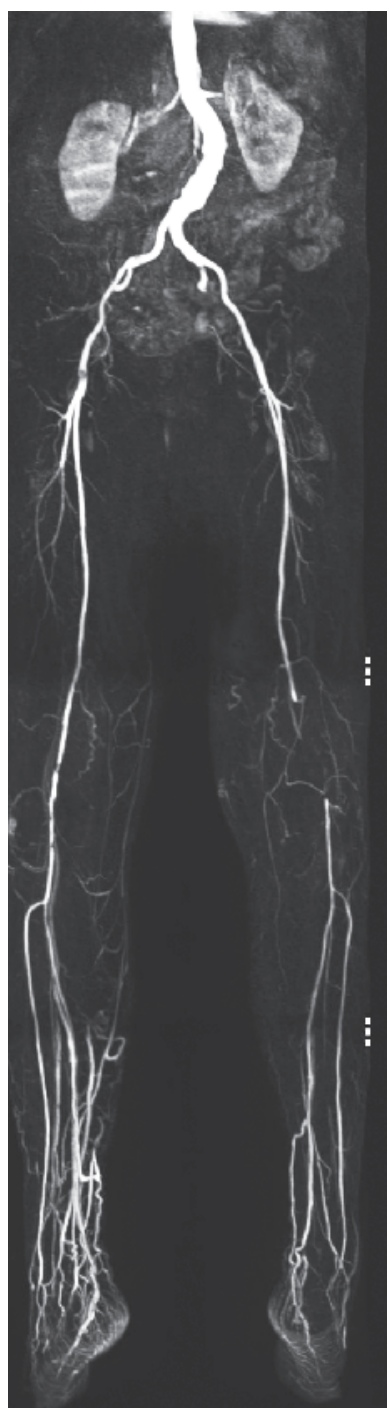

Abb. 2 MR Angiographie mit Verschluss der A. poplitea links
- Stammveneninsuffizienz der V. saphena magna im Stadium I mit Reflux über eine accesorrische Vene

- Stammveneninsuffizienz der Vene bei postthrombotischen Veränderungen mit freiem Abstrom über die Beckenvenen

- Stammveneninsuffizienz der Vene bei Patienten mit pAVK oder KHK

Dies wird in dem nachfolgend geschilderten Fallbericht mehr als ersichtlich.

Fallbericht:

Im September 2017 stellte sich in unserer gefäßchirurgischen Ambulanz notfallmäBig eine 63 Jahre alte Patienten vor. Nach einem Bagatelltrauma im Bereich der linken Großzehe 10 Wochen zuvor, bei dem sie sich diese an einem Schrank gestoßen hatte, kam es nun zur Entwicklung einer Ruheschmerzsymptomatik, verbunden mit einer zunehmenden zyanotischen Veränderung der Zehe. Eine zwischenzeitlich durchgeführte orthopädische Abklärung zeigt keine Fraktur. In der klinischen Untersuchung zeigte sich eine livide verfärbte Großzehe mit einer beginnenden Rötung des Vorfußes ( Abb. 1). Palpatorisch waren lediglich ein Leistenpuls links sowie ein Kniekehlenpuls rechts tastbar. Die Verschlussdrücke der linken Seite waren deutlich reduziert mit einem Knöchel-Arm In$\operatorname{dex}(\mathrm{ABI})$ von 0,5 . Somit zeigte sich bereits in der klinischen Untersuchung eine pAVK im Stadium IV mit einer kritischen Extremitätendurchblutung. Dies bestätigte sich in der weiterführenden bildgebenden Di- agnostik. Duplexsonographisch zeigte sich ein Verschluss der A. poplitea bzw. der distalen A. femoralis superficialis links mit nachgeschaltetem dopplersonographisch lediglich monophasischem Flusssignal, welcher sich in der zusätzlich durchgeführten MR Angiographie bestätigte $(\nabla \mathrm{Abb}$. 2). Zudem zeigte sich in der laborchemischen Untersuchung eine, wenngleich auch geringe, Erhöhung der Entzündungsparameter. In Zusammenschau des klinischen und laborchemischen Befundes wurde eine intravenöse Antibiose mit einem Cephalosporin der dritten Generation eingeleitet und die dringliche Indikation zur Revaskularisation gestellt. Zur Überbrückung des langstreckigen Gefäßverschlusses war die Anlage eines femoro-poplitealen Venenbypasses indiziert.. Bei vorausgegangener Varizenoperation mit Crossektomie und Stripping der VSM war jedoch am linken Bein kein geeignetes Gefäßersatzmaterial vorhanden. Am rechten Bein konnte dagegen eine intakte VSM duplexsonographisch nachgewiesen werden. Diese Vene war 10 Jahre zuvor wegen bekannter Risikofaktoren für die Entwicklung einer pAVK bei Stammveneninsuffizienz im Stadium II nach Hach venenerhaltend mit einer extraluminalen Valvuloplastie operativ rekonstruiert worden. Duplexsonographisch stellte sich die rechte VSM mit einem mittleren Durchmesser von 4,6 $\mathrm{mm}$ und intakter Klappenfunktion dar ( Abb. 3 und 4). Im Rahmen der arteriellen Rekonstruktion einen Tag später, bestätigte sich intraoperativ der hervorragende geeignete Zustand der Vene als Bypassgefäß. Es wurde ein femoro-poplitealer Bypass von der A. femoralis supercialis zur A. poplitea im dritten Poplitealsegment angelegt ( Abb. 5-8). Die Operation verlief komplikationslos. In weiterer Folge kam es zu einem deutlichen Rückgang der zyanotischen Verfärbung der Zehe und der begleitenden Entzündungsreaktion ( Abb. 9). Nach zwei Wochen konnte die Patienten aus der stationären Behandlung entlassen werden und unterzieht sich regelmäßigen Nachkontrollen. Nach einem Nachuntersuchungsintervall von 9 Monaten zeigen sich bisher unauffällige Narbenverhältnisse und eine regelrechte Bypassfunktion. Die Bypassvene weist keine auffälligen Wandveränderungen wie Fibrosierungen oder Aneurysmata auf. 


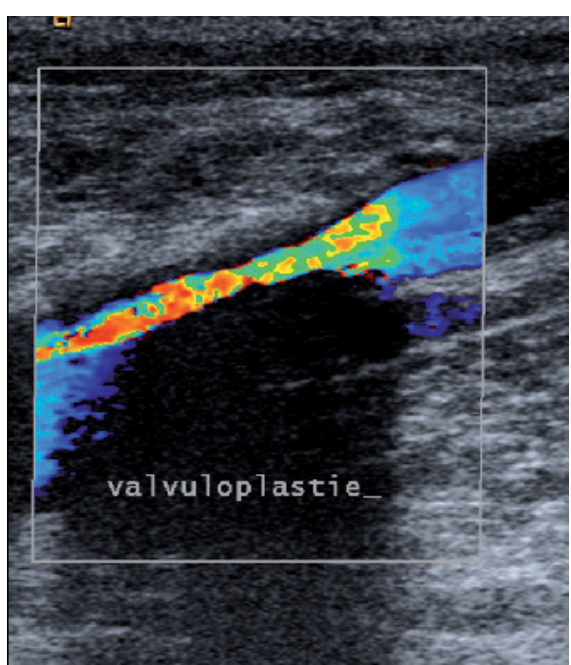

Abb. 3 Sonographie des Venenpatches 10 Jahre postoperativ

\section{Diskussion}

Dies ist einer der ersten beschrieben Fälle einer erfolgreichen Verwendung der VSM im Rahmen einer arteriellen Rekonstruktion nach Durchführung einer extraluminalen Valvuloplastie. Dabei zeigte sich, dass die Vene auch 10 Jahre postoperativ optimal bypassgeeignet war. Intraoperativ zeigten sich in diesem Fall im Bereich der VSM duplexsonographisch und klinisch zudem keine phlebitischen Veränderungen, welche in Langzeitstudien zur extraluminalen Valvuloplastie beschrieben wurden (11). Jedoch fehlen derzeit Langzeitdaten, ob sich Venen nach extraluminaler Valvuloplastie von primär ,gesunden "Venen in ihrem Verhalten als Bypassgefäß unterscheiden. Unabhängig davon bestehen für das Verfahren der extraluminalen Valvuloplastie gute Langzeitdaten hinsichtlich der Therapie der venösen Insuffizienz (1). So zeigte sich beispielsweise bei Joh et al eine signifikante Reduktion des Venendurchmessers sowie eine Retonisierung verbunden mit einer verbesserten venösen Hämodynamik 10 Jahre postoperativ (12). Im Allgmeinen ist daher anzunehmen, dass mit zunehmender Verbreitung der extraluminalen Valvuloplastie in Zukunft weitere Patienten von ihrem eigenen Venenmaterial als mögliches Spendergefäß für autologen Rekonstruktionen profitieren könnten.
Abb. 4

Durchmesser der V. saphena magna 10 Jahre postoperativ

Abb. 5

Präoperative Operationsplanung

Abb. 6

Entnahme der V. saphena magna intraoperativ
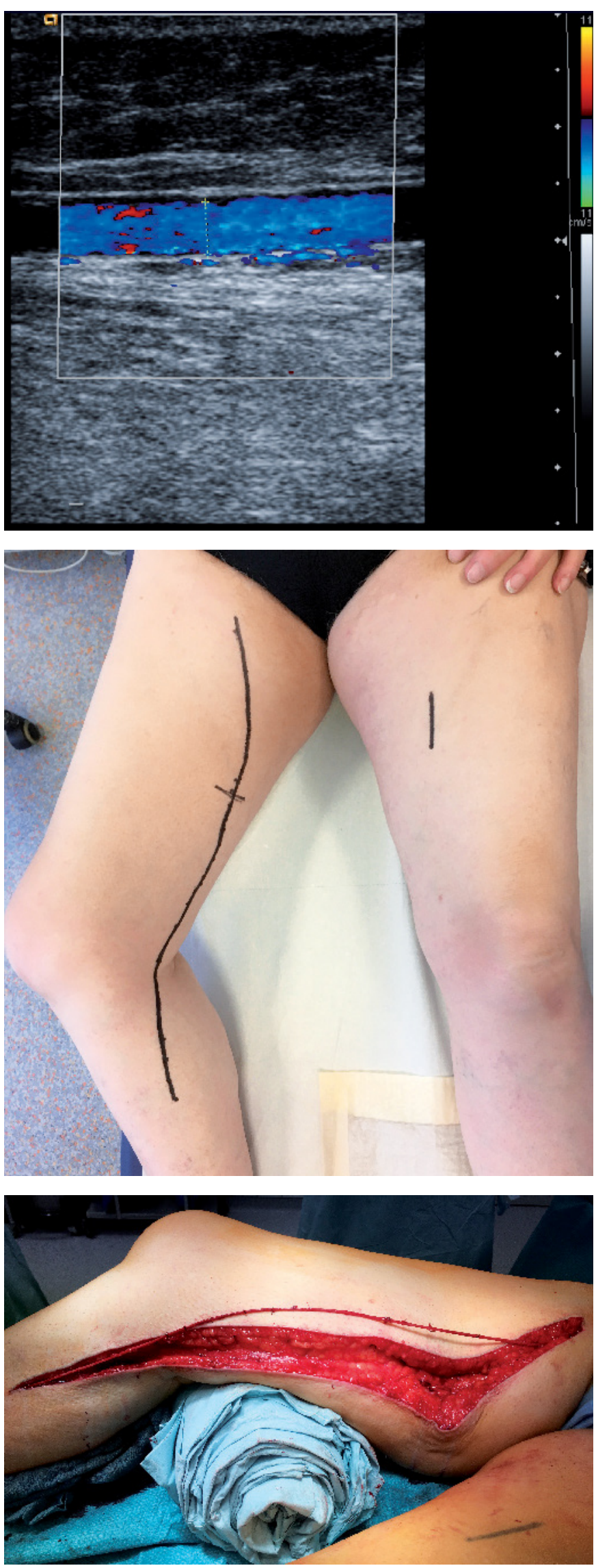


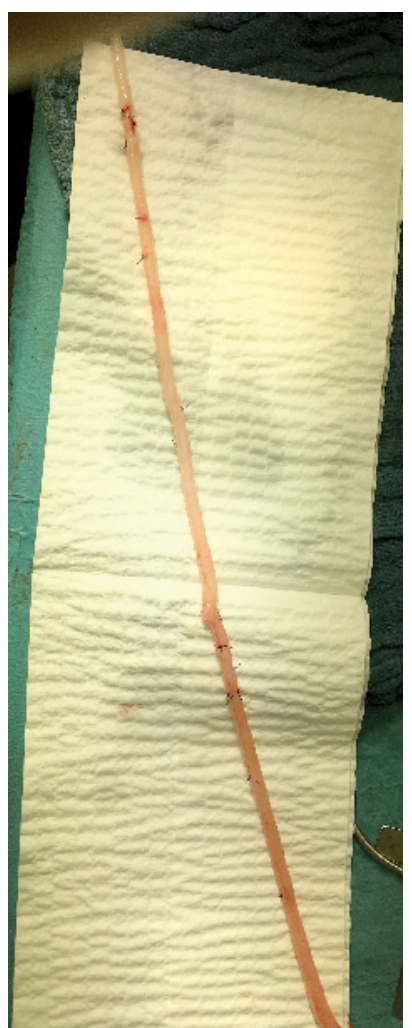

Abb. 7 V. saphena magna im Detail ohne Hinweis auf postphlebitische Veränderungen

\section{Schlussfolgerung}

Bei Patienten mit atherogenen Risikofaktoren sollte bei der Indikationsstellung für die Behandlung einer Stamminsuffizienz der VSM bedacht werden, dass die Vene nach wie vor ein wichtiges Gefäßersatzmaterial in der Herz- und Gefäßchirurgie ist. Mit dem Verfahren der extraluminalen Valvuloplastie kann eine insuffiziente VSM rekonstruktiv und damit venenerhaltend behandelt werden. Im Bedarfsfall steht die rekonstruierte Stammvene als Material für kleinkalibrige Gefäßersatzoperationen zur Verfügung. Die in unserem Fallbeispiel 10 Jahre nach extraluminaler Valvuloplastie zum Gefäßersatz verwendete VSM zeigte im Verlauf keine auffälligen degenerativen Veränderungen.

\section{Interessenkonflikt}

Nach Angaben der Autoren bestehen keine Interessenkonflikte.

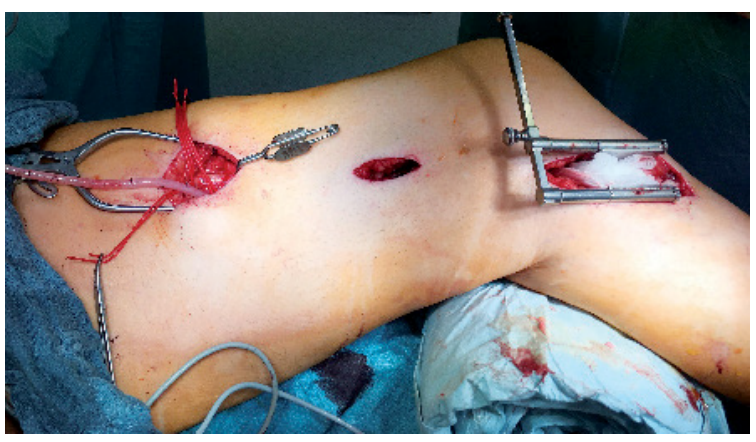

Abb. 8

Arterielle Rekonstruktion

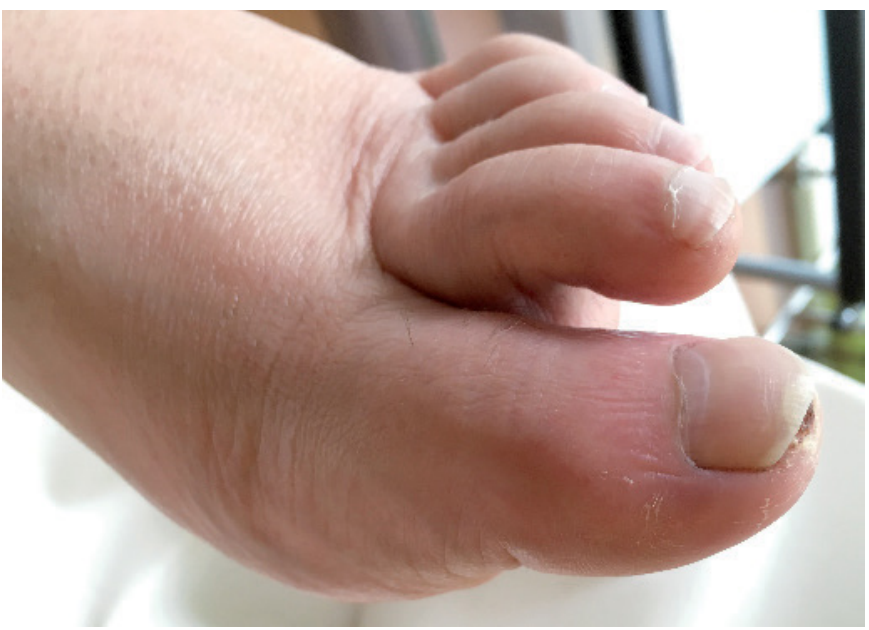

Abb. 9

Postoperatives Ergebniss der Großzehe

\section{Ethische Richtlinien}

Für das Manuskript wurden keine Studien an Menschen oder Tieren durchgeführt.

\section{Literatur}

1. Mumme A, Stücker M, Hummel T. Die extraluminale Vadlvuloplastie der Vena saphena magna. Gefäßchirurgie 2014, 637-642.

2. Hach, Mumme, Hach-Wunderle. Venenchirurgie, 3. Auflage. Stuttgart, Germany: Schattauer 2013.

3. Pittaluga $P$, Chastanet S. Persistent incompetent truncal veins should not be treated immediately. Phlebology 2015; 30(1 Suppl): 98-106.

4. Franceschi $\mathrm{C}$ et al. CHIVA: hemodynamic concept, strategy and results. Int Angiol 2016; 35(1): $8-30$.

5. Lane RJ, Cuzzilla ML, Coroneos JC. The treatment of varicose veins with external stenting to the saphenofemoral junction. Vasc Endovascular Surg, 2002. 36(3): p. 179-92.

6. Lane RJ et al. Recurrence rates following external valvular stenting of the saphenofemoral junction: a comparison with simultaneous contralateral stripping of the great saphenous vein. Eur J Vasc Endovasc Surg 2007; 34(5): 595-603; discussion 604.
7. Onida S, Davies AH. CHIVA, ASVAL and related techniques--Concepts and evidence. Phlebology, 2015; 30(2 Suppl): 42-45.

8. Mühlberger D, Morandini L, Brenner E. Venous valves and major superficial tributary veins near the saphenofemoral junction. J Vasc Surg 2009; 49(6): 1562-1569.

9. Mühlberger D et al. Die extraluminale Vadlvuloplastie. Phlebologie, 2014. 43: 210-212.

10. Almasri $J$ et al. A systematic review and metaanalysis of revascularization outcomes of infrainguinal chronic limb-threatening ischemia. J Vasc Surg, 2018.

11. Sarac A et al. Long-term results of external valvuloplasty in adult patients with isolated great saphenous vein insufficiency. Clin Interv Aging, 2014. 9: p. 575-9.

12. Joh JH et al. External banding valvuloplasty for incompetence of the great saphenous vein: 10-year results. Int J Angiol, 2009. 18(1): 25-28. 